\title{
Scalp recurrence of cholangiocarcinoma after curative hepatectomy: A report of two cases
}

\author{
ENVER KUNDUZ ${ }^{1}$, KURSAT RAHMI SERIN ${ }^{2}$ and MESUT SEKER ${ }^{3}$ \\ ${ }^{1}$ Department of General Surgery, Faculty of Medicine, Bezmialem University, Istanbul 34093; \\ ${ }^{2}$ Bosphorus Clinical Sciences Academy, Istanbul 34357; ${ }^{3}$ Department of Medical Oncology, \\ Faculty of Medicine, Bezmialem University, Istanbul 34093, Turkey
}

Received July 26, 2017; Accepted September 20, 2017

DOI: $10.3892 / \mathrm{mco} .2017 .1467$

\begin{abstract}
Cholangiocarcinomas are the second most common primary liver malignancies. The lymphatics are the common route of metastatic spread for cholangiocarcinomas. The most frequent sites of metastasis are the liver, abdominal lymph nodes, peritoneum and lungs. Cutaneous metastasis of cholangiocarcinoma is rare, and most commonly occurs following percutaneous biliary drainage. Brain or skull metastases from this tumor are uncommon. However, these rare metastatic lesions may occasionally be the first disease manifestation, although scalp recurrence following curative treatment is uncommon. We herein present the cases of two patients with cholangiocarcinoma, who were evaluated as resectable based on the criteria for cholangiocarcinoma resectability, and developed scalp recurrence following curative hepatectomy. Therefore, although scalp recurrence is a rare occurrence following curative treatment for cholangiocarcinoma, metastases should be included in the differential diagnosis.

\section{Introduction}

Cholangiocarcinoma (CC) is the second most common primary liver malignancy (1). CC arises from the biliary ductal epithelium and may be localized in the liver biliary tract (intrahepatic $\mathrm{CC}$ ) or the hepatic hilum (hilar CC). Common presentations of CC include obstructive jaundice, biliary sepsis, liver failure and pain. The treatment goal for $\mathrm{CC}$ is resection with negative histological margins (2). However, despite the advances in radiological diagnostic modalities and interventions, only a minority of the patients are considered eligible for curative surgery at presentation.
\end{abstract}

Correspondence to: Dr Enver Kunduz, Department of General Surgery, Faculty of Medicine, Bezmialem University, Bezmialem Universitesi Hastanesi 4. Kat Genel Cerrahi Sekreterligi, Vatan Caddesi, Fatih, Istanbul 34093, Turkey

E-mail:drkunduz@yahoo.com

Key words: cholangiocarcinoma, hepatectomy, scalp recurrence
The lymphatics are a common route for the metastatic spread of CC, with the most frequent metastatic sites being the liver, abdominal lymph nodes, peritoneum and lungs. Cutaneous metastasis of CC is rare, and reportedly occurs most commonly following percutaneous biliary drainage (3). Brain or skull metastasis of this tumor is uncommon $(4,5)$; however, these rare metastatic lesions may occasionally be the first manifestation of the disease (6), although scalp recurrence following curative treatment is uncommon $(4,7)$.

Patients with $\mathrm{CC}$ are evaluated preoperatively according to their medical performance, radiological findings and future liver remnant. Medically unfit patients, cases with distant metastatic lesions (non-satellite hepatic lesions, lymph node metastases beyond the portal vein or hepatic artery, or distant site or organ metastases), patients with extensive local involvement or inadequate future liver remnant, and cases exhibiting major vascular invasion, are considered to be unresectable. The CC resectability criteria (8) are listed in Table I.

We herein present two cases of CC patients who were evaluated as resectable according to the $\mathrm{CC}$ resectability criteria and developed scalp recurrence following curative hepatectomy.

\section{Case reports}

Case 1. A 64-year-old female patient was admitted to the Department of General Surgery of Liv Hospital (Istanbul, Turkey) with severe right upper quadrant abdominal pain in November 2013. An intrahepatic CC located in the right hepatic lobe was detected and was considered to be resectable on radiological evaluation. Informed consent was obtained from the patient and right hepatectomy was performed. On pathological examination, intrahepatic CC was diagnosed, with a papillary and trabecular pattern growth pattern. The surgical margins were tumor-free. Follow-up without adjuvant treatment was decided by the Tumor Board. Clinical follow-up was performed quarterly. At the 6-month and 1-year follow-up, the thoracic and abdominal computed tomography (CT) evaluations and positron emission tomography (PET)/CT scans were normal. At 17 months postoperatively, the patient was admitted to another hospital with headache and a mass in the scalp. The lesion was localized in the posterior part of the right parietal area, it was hard, immobile and it was sized 
Table I. Criteria of cholangiocarcinoma unresectability.

Periferal or hilar
Distal

Medically unfit patients
Medically unfit patients

Distant metastatic disease

Non-satellite hepatic metastases

Lymph node metastases beyond portal vein, hepatic artery (peripancreatic and celiac axis) distribution

Distant metastases in other organ/sites

Extensive local involvement

Bilateral (or contralateral) involvement of the portal vein (some rarely resectable) hepatic artery, secondary biliary radicals

Inadequate FLR

$<30 \%$ of FLR in a patient with normal parencyhma

$<2$ contiguous segments with adequate portal venous and hepatic arterial inflow, adequate hepatic venous and biliary drainage
Distant metastatic disease

Distant metastasis (liver or other organs)

Lymph node metastases beyond portal vein, hepatic artery (peripancreaticand celiac axis) distribution

Major vascular involvement

Significant portal/superior mesenteric vein

Superior mesenteric artery

Common or proper hepatic artery

FLR, future liver remnant.

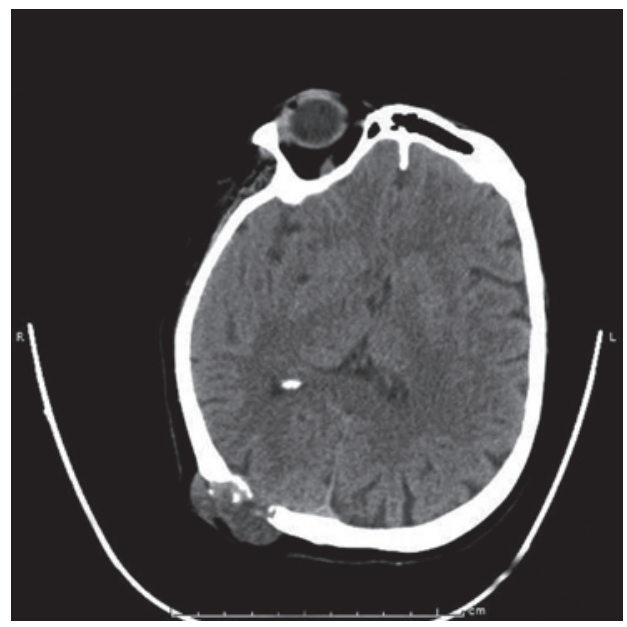

Figure 1. Computed tomography scan of the scalp recurrence from primary cholangiocarcinoma (case 1).

$\sim 2 \times 2 \mathrm{~cm}$. An incisional biopsy was performed by the surgeon and the initial diagnosis was sebaceous cyst. Histopathological evaluation revealed that the scalp lesion was in fact a metastasis from the primary CC. CT scans of the brain, lung and abdomen were performed and the mass was seen invading the skull bone and encroaching on the dura mater (Fig. 1); in addition, multiple lung and iliac crest metastases were detected. A PET-CT scan also revealed involvement of the skull bone by the mass. Radiotherapy at a total dose of 2,500 Gy was delivered to the scalp as palliative therapy. The patient also received adjuvant chemotherapy [6 cycles of gemcitabine and cisplatin and oxaliplatin + leucovorin + 5-fluorouracil (FOLFOX6)] At 43 months after hepatectomy, the patient had a stable skull lesion after radiotherapy. The two lung metastases and one iliac crest metastasis also remained stable. The last follow-up was in June 2017 and the patient remains on capecitabine treatment per os.

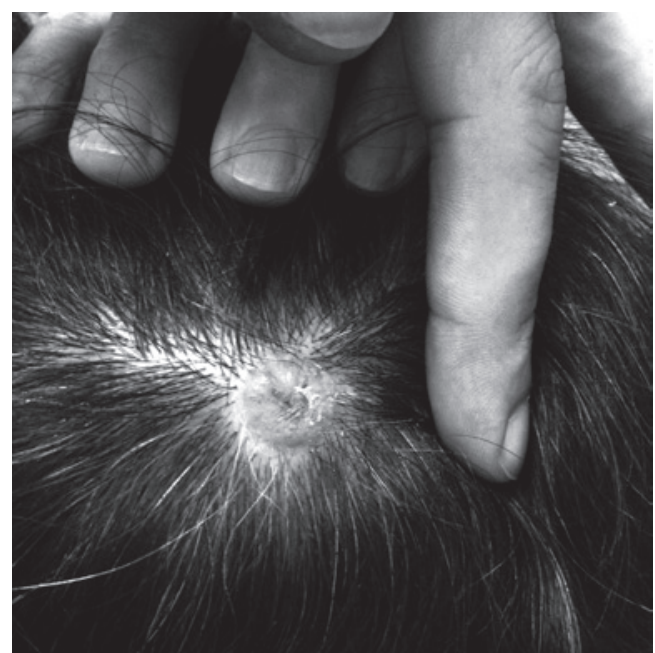

Figure 2. Scalp recurrence from primary cholangiocarcinoma (case 2).

Case 2. A 58-year-old female patient was admitted to the Department of Surgery of Liv Hospital (Istanbul, Turkey) with jaundice in September 2014. Radiological evaluation revealed a hilar CC extending to the left intrahepatic biliary system. Informed consent was obtained from the patient, and left and caudate lobe hepatectomy and periportal lymphadenectomy were performed. $\mathrm{CC}$ was diagnosed following pathological examination of the resected specimen. The surgical margins were tumor-free; however, 5 of the 6 harvested lymph nodes were metastatic. Adjuvant gemcitabine chemotherapy was administered, according to the decision of the Tumor Board. At the 6-month follow-up, a painful scalp mass was detected at the vertex (Fig. 2). The mass was excised with negative surgical margins, and the pathological examination confirmed that it was a metastasis from the primary CC. There were no metastatic lesions on PET/CT. At 30 months after hepatectomy, the patient remained on chemotherapy. At 
the last follow-up (March 2017) there was no disease recurrence.

\section{Discussion}

The majority of metastatic skull lesions originate from breast cancer (9), whereas skull metastasis from CC is a rare entity (4). Skin metastasis of CC may develop following percutaneous transhepatic biliary drainage or along the tract of percutaneous transhepatic cholangiography, and its incidence is $0.6-6 \%(10)$; however, skull metastasis of CC is rarer than skin metastasis.

Skull metastasis may occur either via the lymphatic route or the cerebrospinal venous system (CSVS) $(11,12)$. The CSVS has 2 main divisions: One includes the cortical veins, dural sinuses and cavernous sinuses, and the other is the vertebral venous system, which includes the vertebral venous plexus (Batson plexus) (12). The main characteristic of the Batson plexus is lack of venous valves. This lack of venous valves causes bidirectional blood flow $(11,13)$. The increase in the intra-abdominal pressure leads to retrograde venous flow in the CSVS, and the tumor cells may bypass the lungs and brain and metastasize to the skull (11-13).

Port site seeding during percutaneous transhepatic biliary drainage (PTBD) or percutaneous transhepatic cholangiography (performed as palliative treatment in patients with unresectable disease or to reduce serum bilirubin preoperatively in resectable cases), is a major issue in CC.

A retrospective analysis of 67 patients who underwent PTBD for extrahepatic CC identified 3 patients with catheter tract implantation metastases, presenting as subcutaneous nodules (10). In addition, there have been reports of metastatic seeding at the abdominal wall and peritoneum, chest wall and pleural space, and liver parenchyma, following PTBD (14-16). Sakamoto conducted a prospective study over a 22-year period, in which 7 of 206 (3.4\%) patients who underwent PTBD developed tumor seeding in the PTBD sinus tract as a late complication (17). These findings suggest that the incidence of metastatic tumor seeding along the biliary catheter tract is not as low as initially suspected. A report demonstrated that patients whose bile was positive for tumor cells preoperatively were at higher risk of developing peritoneal metastases (18), whereas a study by Mizuno et al demonstrated that $~ 30-47 \%$ of patients with malignant tumors had tumor cells in the bile (19). Based on these findings, it is likely that metastasis in these patients developed via the hematogenous route, through the valveless Batson venous plexus.

Due to the lack of large case series on the treatment of skull metastasis of CC, there is currently no established treatment protocol for skull metastasis from primary CC. However, there are four treatment modalities used for patients with skull metastases: Surgery, irradiation, chemotherapy and hormonal therapy (4). We believe that, as the main treatment for CC is surgical resection with negative margins, isolated skull metastasis should be resected with clear margins whenever possible.

In conclusion, in $\mathrm{CC}$ patients with scalp lesions, skull metastasis from the primary $\mathrm{CC}$ should be included in the differential diagnosis and the patient should be evaluated with cranial CT and PET/CT scans. Written consent for the publication of the case details was obtained from the patients.

\section{References}

1. Bartella I and Dufour JF: Clinical diagnosis and staging of intrahepatic cholangiocarcinoma. J Gastrointestin Liver Dis 24: 481-489, 2015.

2. Lafaro K, Grandhi MS, Herman JM and Pawlik TM: The importance of surgical margins in primary malignancies of the liver. J Surg Oncol 113: 296-303, 2016.

3. Rosich-Medina A, Liau SS, Jah A, Huguet E, See TC, Jamieson N and Praseedom R: Cutaneous metastases from cholangiocarcinoma following percutaneous transhepatic biliary drainage: Case report and literature review. Int J Surg Case Rep 1: 33-36, 2010.

4. Fujimoto K, Kuroda J, Makino K, Hasegawa Y and Kuratsu J: Skull metastasis from intrahepatic cholangiocarcinoma: Report of 3 cases and review of the literature. Neurol Med Chir (Tokyo) 53: 717-721, 2013.

5. Okamura Y, Harada A, Maeda A, Fujioka A, Horiba T, Ishigure $\mathrm{K}$, Hirai A, Ito $\mathrm{Y}$ and Uesaka $\mathrm{K}$ : Carcinomatous meningitis secondary to cholangiocarcinoma without other systemic metastasis. J Hepatobiliary Pancreat Surg 15: 237-239, 2008.

6. Hyun SY, Lee JH, Shin HS, Lee SW, Park YN and Park JY: Cutaneous metastasis from cholangiocarcinoma as the first clinical sign: A report of two cases. Gut Liver 5: 100-104, 2011.

7. Miyamoto J, Tatsuzawa K, Sasajima H and Mineura K: Metastatic skull tumor from cholangiocarcinoma. Case report. Neurol Med Chir (Tokyo) 47: 132-135, 2007.

8. Schulick RD: Criteria of unresectability and the decision-making process. HPB (Oxford) 10: 122-125, 2008.

9. Mitsuya K, Nakasu Y, Horiguchi S, Harada H, Nishimura T, Yuen S, Asakura K and Endo M: Metastatic skull tumors: MRI features and a new conventional classification. J Neurooncol 104: 239-245, 2011

10. Sakata J, Shirai Y, Wakai T, Nomura T, Sakata E and Hatakeyama K: Catheter tract implantation metastases associated with percutaneous biliary drainage for extrahepatic cholangiocarcinoma. World J Gastroenterol 11: 7024-7027, 2005.

11. Batson OV: The function of the vertebral veins and their role in the spread of metastases. Ann Surg 112: 138-149, 1940.

12. Tobinick E and Vega CP: The cerebrospinal venous system: Anatomy, physiology, and clinical implications. Med Gen Med 8: 53, 2006.

13. Coman DR and deLONG RP: The role of the vertebral venous system in the metastasis of cancer to the spinal column; experiments with tumor-cell suspensions in rats and rabbits. Cancer 4: 610-618, 1951.

14. Miller GA Jr, Heaston DK, Moore AV Jr, Mills SR and Dunnick NR: Peritoneal seeding of cholangiocarcinoma in patients with percutaneous biliary drainage. AJR Am J Roentgenol 141: 561-562, 1983.

15. Uenishi T, Hirohashi K, Inoue K, Tanaka H, Kubo S, Shuto T, Yamamoto T, Kaneko M and Kinoshita H: Pleural dissemination as a complication of preoperative percutaneous transhepatic biliary drainage for hilar cholangiocarcinoma: Report of a case. Surg Today 31: 174-176, 2001.

16. Shimizu Y, Yasui K, Kato T, Yamamura Y, Hirai T, Kodera Y, Kanemitsu Y, Ito S, Shibata N, Yamao K and Ohhashi K: Implantation metastasis along the percutaneous transhepatic biliary drainage sinus tract. Hepatogastroenterology 51: 365-367, 2004.

17. Sakamoto E, Hayakawa N, Kamiya J, Kondo S, Nagino M, Kanai M, Miyachi M, Uesaka K and Nimura Y: Treatment strategy for mucin-producing intrahepatic cholangiocarcinoma: Value of percutaneous transhepatic biliary drainage and cholangioscopy. World J Surg 23: 1038-1044, 1999.

18. Tanaka N, Nobori M and Suzuki Y: Does bile spillage during an operation present a risk for peritoneal metastasis in bile duct carcinoma? Surg Today 27: 1010-1014, 1997.

19. Mizuno T, Ishizaki Y, Komuro Y, Yoshimoto J, Sugo H, Miwa K and Kawasaki S: Surgical treatment of abdominal wall tumor seeding after percutaneous transhepatic biliary drainage. Am J Surg 193: 511-513, 2007. 